\title{
Application of Maximum Entropy/Optimal Projection Design Synthesis to a Benchmark Problem
}

\author{
Emmanuel G. Collins Jr.* and James A. King $\dagger$ \\ Harris Corporation, Melbourne, Florida 32901 \\ and \\ Dennis S. Bernstein $\ddagger$ \\ University of Michigan, Ann Arbor, Michigan 48109
}

\begin{abstract}
Maximum entropy/optimal projection design synthesis is a methodology for designing robust, fixed-order controllers for flexible structures. This paper reviews the theoretical basis for this method and illustrates the approach using a benchmark problem. The benchmark problem involves two masses with spring coupling, an uncertain spring constant, and a sensor and actuator that are noncollocated. The results of this paper also illustrate the use of a precompensation methodology that allows the control designer to precondition the design plant by embedding judiciously designed filters. These filters are included in the implemented controller.
\end{abstract}

\section{Introduction}

A CTIVE feedback control for vibration suppression in lightly damped structures continues to be a challenging area of aerospace engineering.' Typically, such problems involve multi-input/multi-output systems in noncollocated sensor/actuator configurations. The task of designing disturbance attenuation feedback controllers for such systems is further exacerbated by constraints on real-time processing capacity for feedback control law implementation as well as modeling uncertainty associated with complex structures. Among the numerous methodologies proposed for addressing the structural control problem, this paper focuses on the maximum entropy/optimal projection (ME/OP) approach. This approach was originally developed by Hyland ${ }^{2-5}$ and Hyland and Madiwale ${ }^{6}$ in a series of technical reports and conference papers. The ME/OP approach has been applied experimentally to various structural control testbeds ${ }^{7-9}$ and has been evaluated by other researchers in Refs. 10-12. Subsequently, numerous extensions and variations of this approach have been developed to address a variety of problems in robust, fixed-structure controller synthesis. ${ }^{13-17}$ A detailed review of this work with extensive references to related literature is given in Ref. 18.

The purpose of this paper is twofold. First we provide (in Secs. II and III) a brief review of robust fixed-order controller synthesis, in particular, robust optimal projection controller synthesis. Although a detailed review is given in Ref. 18, the brief review given here focuses more directly on the ME/OP technique for robust controller synthesis, which is described in Sec. III. After reviewing ME/OP, we then turn our attention in Sec. IV to problems 1 and 2 of the benchmark example given in Ref. 19. The ME/OP design approach is applied to the benchmark example in Sec. V, and the design results are discussed in Sec. VI. A conclusion is presented in Sec. VII.

\section{Robust Fixed-Order Controller Synthesis}

Optimal projection theory ${ }^{13}$ generalizes linear quadratic Gaussian (LQG) theory to the case of reduced-order con-

Received May 1, 1991; revision received Dec. 4, 1991; accepted for publication Feb. 4, 1992. Copyright (C) 1992 by the American Institute of Aeronautics and Astronautics, Inc. All rights reserved.

*Associate Principal Engineer, Government Aerospace Systems Division. Member AIAA.

$\dagger$ Senior Engineer, Government Aerospace Systems Division.

$\ddagger$ Associate Professor, Department of Aerospace Engineering. trollers. Although LQG theory provides quadratically $\left(\mathrm{H}_{2}\right)$ optimal full-order dynamic compensators by means of two uncoupled Riccati equations, optimal projection theory characterizes quadratically optimal reduced-order (i.e., fixed-order) controllers via a coupled system consisting of two modified Riccati equations and two modified Lyapunov equations. When the controller order is set equal to the plant order, the projection matrix $\tau$ responsible for the coupling (the so-called "optimal projection") becomes the identity, the coupling terms vanish, the Lyapunov equations are rendered superfluous, and the LQG Riccati equations are recovered. Numerical algorithms for solving the optimal projection equations via iterative and homotopy techniques are discused in Refs. $20-22$.

Robust optimal projection theory (as well as robust LQG theory) has been developed by incorporating uncertainty bounds within the design optimization procedure. The idea behind this approach can most clearly be illustrated within the context of robust analysis, whereas its application to synthesis is a fairly straightforward extension of fixed-structure optimization. The description given here follows the development of uncertainty bounds given in Ref. 23 .

For the asymptotically stable linear system

$$
\dot{x}(t)=A x(t)
$$

we consider a quadratic Lyapunov function of the form

$$
\mathrm{N}(x)=x^{T} P x
$$

where the positive-definite matrix $P$ is given by the Lyapunov equation

$$
0=A^{T} P+P A+R
$$

where $R$ is positive definite. To address additive disturbances for a system of the form

$$
\dot{x}(t)=A x(t)+w(t)
$$

it is convenient to utilize the dual equation

$$
0=A Q+Q A^{T}+V
$$

in which $A$ is replaced by $A^{T}$ (which has the same spectrum as $A$ ) and where $V$ is interpreted as the intensity of the white noise disturbance $w(t)$. In Eq. (5), the matrix $Q$ can be viewed 
as a controllability Gramian or covariance matrix with associated quadratic $\left(\mathrm{H}_{2}\right)$ performance measure

$$
J=\operatorname{tr} Q R=\operatorname{tr} P V
$$

If $A$ is uncertain so that Eq. (1) is replaced by

$$
\dot{x}(t)=(A+\Delta A) x(t)
$$

where $\Delta A \in \mathcal{U}$, a set of perturbations, then we wish to determine whether $A+\Delta A$ remains stable for all $\Delta A \in \mathcal{U}$. One approach to this problem involves replacing Eq. (5) by

$$
0=A Q+Q A^{T}+\Omega+V
$$

where $\Omega$ is a constant positive-definite matrix. Rewriting Eq. (8) as

$$
0=(A+\Delta A) Q+Q(A+\Delta A)^{T}+\Omega-\left(\Delta A Q+Q \Delta A^{T}\right)+V
$$

it follows that $A+\Delta A$ is stable so long as $\Delta A$ satisfies

$$
\Delta A Q+Q \Delta A^{T} \leq \Omega
$$

where $Q$ is the solution to Eq. (8).

A variation on Eq. (8) involves allowing $\Omega$ to be a function of $Q$. Thus we consider the modified Lyapunov equation

$$
0=A Q+Q A^{T}+\Omega(Q)+V
$$

where $\Omega(\cdot)$ satisfies

$$
\Delta A Q+Q \Delta A^{T} \leq \Omega(Q), \quad \text { for all } \Delta A \in \mathcal{U}
$$

and for all non-negative-definite $Q$. It then follows by rewriting Eq. (11) as

$$
\begin{aligned}
0= & (A+\Delta A) Q+Q(A+\Delta A)^{T}+\Omega(Q) \\
& -\left(\Delta A Q+Q \Delta A^{T}\right)+V
\end{aligned}
$$

that $A+\Delta A$ is stable. Furthermore, letting $Q_{\Delta A}$ satisfy

$$
0=(A+\Delta A) Q_{\Delta A}+Q_{\Delta A}(A+\Delta A)^{T}+V
$$

and subtracting Eq. (14) from Eq. (13) yields

$$
\begin{aligned}
0 & =(A+\Delta A)\left(Q-Q_{\Delta A}\right)+\left(Q-Q_{\Delta A}\right)(A+\Delta A)^{T} \\
& +\Omega(Q)-\left(\Delta A Q+Q \Delta A^{T}\right)
\end{aligned}
$$

which implies that

$$
Q_{\Delta A} \leq Q, \quad \Delta A \in \mathcal{U}
$$

Thus $\operatorname{tr}(Q R)$ provides a worst-case bound for the actual $\mathrm{H}_{2}$ performance $\operatorname{tr}\left(Q_{\triangle A} R\right)$.

Since the ordering induced by the cone of non-negative-definite matrices is only a partial ordering, it should not be expected that there exists an operator $\Omega(\cdot)$ satisfying Eq. (12) that is a least upper bound. Indeed, there are many alternative definitions for the bound $\Omega(\cdot)$. To illustrate some of these alternatives, assume for convenience that $\Delta A$ is of the form

$$
\Delta A=\sigma_{1} A_{1}, \quad\left|\sigma_{1}\right| \leq \delta_{1}
$$

where $\sigma_{1}$ is an uncertain real scalar parameter assumed only to satisfy the stated bounds and $A_{1}$ is a known matrix denoting the structure of the parametric uncertainty. The bound $\Omega(\cdot)$ utilized in Ref. 24 for full-state-feedback design was chosen to be the absolute value bound

$$
\Omega(Q)=\delta_{1}\left|A_{1} Q+Q A_{1}^{T}\right|
$$

where $|\cdot|$ denotes the non-negative-definite matrix obtained by replacing each eigenvalue by its absolute value. Since the bound defined in Eq. (18) is not differentiable with respect to $Q$, it has limited usefulness in fixed-structure controller synthesis. A more useful bound is the quadratic (in $Q$ ) bound

$$
\Omega(Q)=\delta_{1}\left(A_{L} A_{L}^{T}+Q A_{R}^{T} A_{R} Q\right)
$$

which has been considered in Refs. 25 and 26. In Eq. (19), $A_{L}$ and $A_{R}$ are a factorization of $A_{1}$ of the form $A_{1}=A_{L} A_{R}$. A third bound that has been considered is the linear (in $Q$ ) bound

$$
\Omega(Q)=\delta_{1}\left(\alpha Q+\alpha^{-1} A_{1} Q A_{1}^{T}\right)
$$

where $\alpha$ is an arbitrary positive scalar. As discussed in Ref. 14, the linear bound is closely related to a multiplicative white noise model.

Within the context of fixed-structure controller synthesis, the linear and quadratic bounds have been merged with optimal projection theory in Refs. 15 and 16, respectively. The quadratic bound also has the useful property that it enforces an $H_{\infty}$ (bounded real) constraint. This extension has been incorporated within optimal projection theory in Ref. 17.

In summary, it can be seen that both the linear and quadratic bounds guarantee robust stability and performance with respect to parameter uncertainty and lead to generalizations of LQG and optimal projection theory. As discussed in Ref. 27, however, these bounds actually guarantee robustness with respect to time-varying parameter variations, which may lead to conservatism when the parameter variations are known to be constant. Viewed in the frequency domain, such bounds correspond to small-gain-type conditions that enforce robust stability with respect to complex, frequency-dependent uncertainty, which is conservative if the uncertain parameters are known to be real and constant. Such conservatism may have serious consequences in controlling flexible structures with stiffness uncertainty, which is a highly structured, inherently real form of parameter uncertainty. Consequently, we now turn our attention to the maximum entropy/optimal projection approach to robust controller synthesis that seeks to overcome these difficulties for a particular form of parametric uncertainty.

\section{Maximum Entropy/Optimal Projection Design Synthesis}

As discussed in Sec. I, the ME/OP approach was originally developed in Refs. 2-6. In brief, the basis of the ME/OP idea is to choose the operator $\Omega(Q)$ in the modified Lyapunov equation (8) to be of the form

$$
\Omega(Q)=\sum_{i=1}^{r} \delta_{i}\left(1 / 2 A_{i}^{2} Q+A_{i} Q A_{i}^{T}+1 / 2 Q A_{i}^{2 T}\right)
$$

where the summation corresponds to an uncertainty model of the form $A+\Delta A$, where

$$
\Delta A=\sum_{i=1}^{r} \sigma_{i} A_{i}
$$

where $\sigma_{1}, \ldots, \sigma_{r}$ are uncertain real parameters, and $\delta_{1}, \ldots, \delta_{r}$ $\geq 0$ are uncertainty scalings. Note that in Eq. (17) we set $r=1$ for convenience, although Eqs. (17-20) could readily be generalized to the case $r>1$.

The unusual feature of Eq. (21) is that (as will be seen shortly) $\Omega(Q)$ is not a bound in the sense of Eq. (12) as are Eqs. (18-20). Thus we do not stipulate a precise uncertainty range for the uncertain parameters $\sigma_{i}$ as in Eq. (17). Rather, the constants $\delta_{1}, \ldots, \delta_{r}$ should only be viewed as scalings. Indeed, whereas the bounds of Eqs. (18-20) are valid for arbitrary choices of $A_{1}$, the operator in Eq. (21) will only be used (in this paper) under restrictive, but practically useful, assumptions. Specifically, we now assume that the nominal 
dynamics matrix $A$ is of the form

$$
A=\text { block-diag }\left(\left[\begin{array}{cc}
-\eta_{1} & \omega_{1} \\
-\omega_{1} & -\eta_{1}
\end{array}\right], \ldots,\left[\begin{array}{cc}
-\eta_{r} & \omega_{r} \\
-\omega_{r} & -\eta_{r}
\end{array}\right]\right)
$$

which is representative of a lightly damped structure in a modal basis, whereas the uncertainty matrix $A_{i}$ is of the form

$$
A_{i}=\text { block-diag }\left(0, \ldots, 0,\left[\begin{array}{cc}
0 & 1 \\
-1 & 0
\end{array}\right], 0, \ldots, 0\right)
$$

where the position of the matrix

$$
\left[\begin{array}{cc}
0 & 1 \\
-1 & 0
\end{array}\right]
$$

corresponds to the $i$ th diagonal block of $A$. Since the poles of $A+\Sigma_{i=1}^{r} \sigma_{i} A_{i}$ are of the form $-\eta+j\left(\omega_{i}+\sigma_{i}\right)$, each term $\sigma_{i} A_{i}$ represents uncertainty in the imaginary part of a pole location.

To further illustrate the structure of $\Omega(Q)$ given by Eq. (21), define

$$
S \triangleq \sum_{i=1}^{r} 1 / 2 \delta_{i} A_{i}^{2}
$$

so that Eq. (21) becomes

$$
\Omega(Q)=S Q+Q S+\sum_{i=1}^{r} \delta_{i} A_{i} Q A_{i}^{T}
$$

and the modified Lyapunov equation (11) is of the form

$$
0=(A+S) Q+Q(A+S)^{T}+\sum_{i=1}^{r} \delta_{i} A_{i} Q A_{i}^{T}+V
$$

Using Eq. (24) and the fact that

$$
\left[\begin{array}{cc}
0 & 1 \\
-1 & 0
\end{array}\right]^{2}=\left[\begin{array}{cc}
-1 & 0 \\
0 & -1
\end{array}\right]
$$

$S$ can be written as

$$
S=\text { block-diag }\left(\left[\begin{array}{cc}
-1 / 2 \delta_{1} & 0 \\
0 & -1 / 2 \delta_{1}
\end{array}\right], \ldots,\left[\begin{array}{cc}
-1 / 2 \delta_{r} & 0 \\
0 & -1 / 2 \delta_{r}
\end{array}\right]\right)
$$

It is interesting to contrast Eq. (26) with the linear bound of Eq. (20) in light of the structure of $S$. To do this, generalize Eq. (20) to the case $r>1$ (but setting $\alpha=1$ ), which now has the form

$$
\Omega(Q)=\sum_{i=1}^{r} \delta_{i}\left(Q+A_{i} Q A_{i}^{T}\right)
$$

Now rewrite Eq. (29) as

$$
\Omega(Q)=\hat{S} Q+Q \hat{S}+\sum_{i=1}^{r} \delta_{i} A_{i} Q A_{i}^{T}
$$

where

$$
S \triangleq 1 / 2 \sum_{i=1}^{r} \delta_{i} I
$$

which yields a modified Lyapunov equation of the form

$$
0=(A+\hat{S}) Q+Q(A+\hat{S})^{T}+\sum_{i=1}^{r} \delta_{i} A_{i} Q A_{i}^{T}+V
$$

which is identical to Eq. (27) with $S$ replaced by $\hat{S}$.

Maximum entropy design appears to be closely related to recent results on parameter-dependent Lyapunov functions and variations of the Popov criteria. ${ }^{28}$ In Ref. 29 , it is shown that the maximum entropy covariance equation (31) describes the average covariance for a Cauchy uncertainty probability distribution.

Given the modified Lyapunov equation (27), the ME/OP design equations can be derived in a straightforward manner following the technique given in Ref. 15 . Hence, consider the nominal plant

$$
\begin{gathered}
\dot{x}=A x+B u+D_{1} w_{1}, \quad x \in \boldsymbol{R}^{n_{s}}, \quad u \in \boldsymbol{R}^{n_{u}}, \quad w_{1} \in \boldsymbol{R}^{n_{w_{1}}} \\
y=C x+w_{2}, \quad y \in \boldsymbol{R}^{n_{y}} \\
z=E_{1} x, \quad z \in \boldsymbol{R}^{n_{s}}
\end{gathered}
$$

where $y$ is the sensor output, $z$ is the performance variable, and $w_{1}$ and $w_{2}$ are (for convenience only) uncorrelated, white noise disturbances with intensities $V_{1} \geq 0$ and $V_{2}>0$, respectively. Also, consider the $\mathrm{H}_{2}$ cost functional

$$
J\left(A_{c}, B_{c}, C_{c}\right)=\lim _{t \rightarrow \infty} E\left(x^{T} R_{1} x+u^{T} R_{2} u\right)
$$

where $R_{1}=E_{1}^{T} E_{1}$ and $R_{2}>0$. The matrices $A_{c}, B_{c}$, and $C_{c}$ characterize the $n_{c}$ th-order dynamic compensator $\left(n_{c} \leq n\right)$

$$
\begin{gathered}
\dot{x}_{c}=A_{c} x_{c}+B_{c} y, \quad x_{c} \in R^{n_{c}} \\
u=-C_{c} x_{c}
\end{gathered}
$$

Optimization of the performance functional (35) with the modified covariance model (27) applied to the closed-loop system yields dynamic compensator gains

$$
\begin{gathered}
A_{c}=\Gamma\left(A_{s}-Q \bar{\Sigma}-\Sigma P\right) G^{T} \\
B_{c}=\Gamma Q C^{T} V_{2}^{-1} \\
C_{c}=R_{2}^{-1} B^{T} P G^{T}
\end{gathered}
$$

where the $n \times n$ non-negative-definite matrices $Q, P, \hat{Q}$, and $\hat{P}$ satisfy

$$
\begin{aligned}
& 0= A_{s} Q+Q A_{s}^{T}+\bar{V}_{1}+\sum_{i=1}^{r} \delta_{i} A_{i}(Q+\hat{Q}) A_{i}^{T}-Q \bar{\Sigma} Q \\
&+\tau_{\perp} Q \bar{\Sigma} Q \tau_{\perp}^{T} \\
& 0= A_{s}^{T} P+P A_{s}+R_{1}+\sum_{i=1}^{r} \delta_{i} A_{i}^{T}(P+\hat{P}) A_{i}-P \Sigma P \\
&+\tau_{\perp}^{T} P \Sigma P \tau_{\perp} \\
& 0=\left(A_{s}-\Sigma P\right) \hat{Q}+\hat{Q}\left(A_{s}-\Sigma P\right)^{T}+Q \bar{\Sigma} Q-\tau_{\perp} Q \bar{\Sigma} Q \tau_{\perp}^{T} \\
& 0=\left(A_{s}-Q \bar{\Sigma}\right)^{T} \hat{P}+\hat{P}\left(A_{s}-Q \bar{\Sigma}\right)+P \Sigma P-\tau_{\perp}^{T} P \Sigma P \tau_{\perp} \\
& \quad \operatorname{rank} \hat{Q}=\operatorname{rank} \hat{P}=\operatorname{rank} \hat{Q} \hat{P}=n_{c} \\
& \quad \tau=\hat{Q} \hat{P}(\hat{Q} \hat{P})^{\#}
\end{aligned}
$$

where $(\cdot)^{\sharp}$ denotes the group generalized inverse and $\tau$ has the factorization

$$
\begin{gathered}
\tau=G^{T} \Gamma, \quad G, \Gamma \in R^{n_{c} \times n} \\
A_{s} \triangleq A+\sum_{i=1}^{r} 1 / 2 \delta_{i} A_{i}^{2}, \quad \Sigma \triangleq B R_{2}^{-1} B^{T} \\
\bar{\Sigma} \triangleq C^{T} V_{2}^{-1} C, \quad \bar{V}_{1} \triangleq D_{1} V_{1} D_{1}^{T}
\end{gathered}
$$

The matrix $\tau$ defined in Eq. (46) is an oblique projection matrix (the optimal projection) that is responsible for enforcing the reduced-order constraint $n_{c} \leq n$ on the compensator. 
The ME/OP design equations (41-46) can be solved by using a homotopy algorithm. ${ }^{21,30}$ As illustrated by Fig. 1, this homotopy algorithm allows the deformation of an LQG controller into a full-order maximum entropy controller. The maximum entropy controller is then reduced to an appropriate order by using an indirect controller reduction method. It is important that this initial reduced-order controller approximately solves the ME/OP design equations to within a small error, although it is not required to be a stabilizing controller. This can be achieved by beginning with a low authority LQG design and/or incorporating a sufficiently high level of uncertainty in the ME design. In practice a slight modification of the balanced controller reduction algorithm of Yousuff and Skelton ${ }^{31}$ is currently used as the indirect controller method. Once this initial reduced-order controller is obtained, the homotopy algorithm is used to deform this controller into an ME/OP controller. Then, if a higher authority controller is desired, the final step of the algorithm is to increase the controller authority to a desirable level.

\section{Benchmark Problem}

We now turn our attention to the benchmark problem of Ref. 19. The dynamical system is described, and two associated design problems are presented.

Consider the two-mass/spring system shown in Fig. 2, which is a generic model of an uncertain dynamical system with a noncollocated sensor and actuator pair. A control force acts on body 1, and the position of body 2 is measured, resulting in a noncollocated control problem. This system can be represented in state-space form as

$$
\begin{aligned}
& {\left[\begin{array}{l}
\dot{x}_{1} \\
\dot{x}_{2} \\
\dot{x}_{3} \\
\dot{x}_{4}
\end{array}\right]=\left[\begin{array}{cccc}
0 & 0 & 1 & 0 \\
0 & 0 & 0 & 1 \\
-k / m_{1} & k / m_{1} & 0 & 0 \\
k / m_{2} & -k / m_{2} & 0 & 0
\end{array}\right]\left[\begin{array}{l}
x_{1} \\
x_{2} \\
x_{3} \\
x_{4}
\end{array}\right]} \\
& +\left[\begin{array}{c}
0 \\
0 \\
1 / m_{1} \\
0
\end{array}\right] u+\left[\begin{array}{c}
0 \\
0 \\
0 \\
1 / m_{2}
\end{array}\right] w_{1}
\end{aligned}
$$

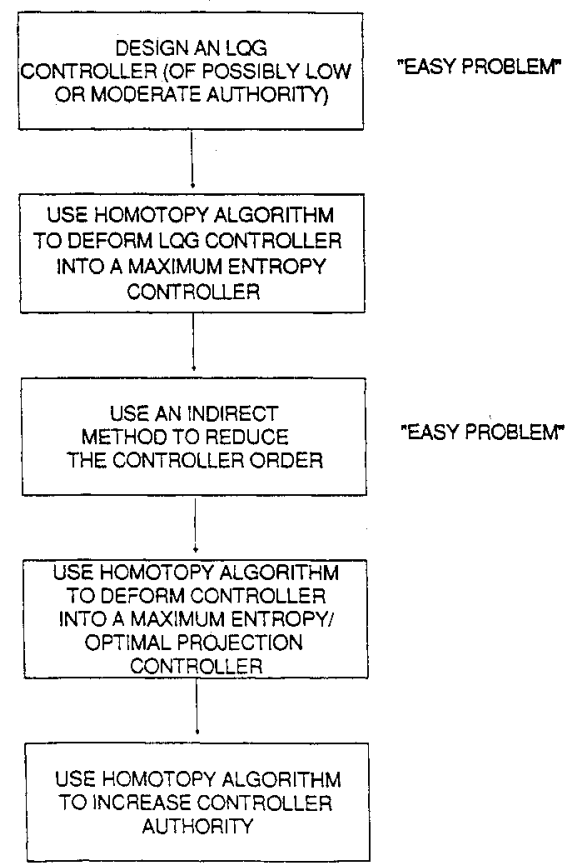

Fig. 1 Practical application of the maximum entropy/optimal projection control-design algorithm.

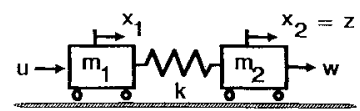

Fig. 2 Benchmark system.

where

$$
y^{\prime}=z=x_{2}, \quad y=y^{\prime}+v
$$

$x_{1}=$ position of body 1

$x_{2}=$ position of body 2

$x_{3}=$ velocity of body 1

$x_{4}=$ velocity of body 2

$u=$ control input

$w_{1}=$ plant disturbance

$z=$ performance variable (output to be controlled)

$y^{\prime}=$ noise-free measurement

$v=$ sensor noise

\section{Design Problems}

Design Problem 1 form

Design a constant gain linear feedback compensator of the

$$
\begin{aligned}
& \dot{x}=A_{c} x_{c}+B_{c} y \\
& u=C_{c} x_{c}+D_{c} y
\end{aligned}
$$

(any of these matrices may of course be zero) with the following properties:

1) The closed-loop system is stable for $m_{1}=m_{2}=1$ and $0.5<k<2.0$.

2) For $w(t)=$ unit impulse at $t=0$, the performance variable $z$ has a settling time of about $15 \mathrm{~s}$ for the nominal system $m_{1}=m_{2}=k=1$.

3) The control system can tolerate reasonable measurement noise signals $v(t)$.

4) Achieve reasonable performance/stability robustness with reasonable bandwidth.

5) Use reasonable controller effort.

6) Use reasonable controller complexity.

Design Problem 2

Same as design problem 1 except in place of step 2 insert the following:

The $w(t)$ is a sinusoidal disturbance of frequency $0.5 \mathrm{rad} / \mathrm{s}$ whose amplitude and phase, although constant, are not available to the designer. Achieve asymptotic rejection of $w(t)$ at the performance variable $z(t)$ [i.e., minimize $\lim \sup _{t \rightarrow \infty} z(t)$ with a 20-s settling time] for $m_{1}=m_{2}=1,0.5<k<2.0$.

\section{ME/OP Control Design for the Benchmark Problem}

This section considers design problems 1 and 2 of the benchmark problem described in the previous section. In particular, the development of robust controllers using the ME/OP approach is described. This approach was first applied to the benchmark problem in Refs. 32 and 33.

We begin by introducing some notation. Consider the plant

$$
\begin{gathered}
\dot{x}(t)=A x(t)+B u(t)+D_{1} w_{1}(t) \\
z(t)=E_{1} x(t) \\
y^{\prime}(t)=C x(t)
\end{gathered}
$$

Then $G(s)$ is said to be the transfer matrix representation of Eqs. (53-55) if

$$
\left[\begin{array}{c}
z(s) \\
y^{\prime}(s)
\end{array}\right]=[G(s)]\left[\begin{array}{l}
w_{1}(s) \\
u(s)
\end{array}\right]
$$




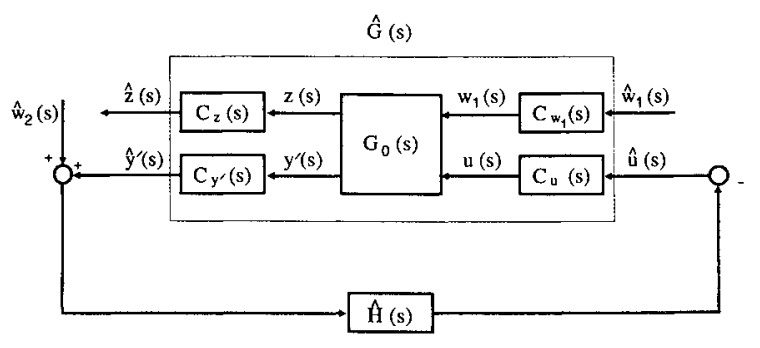

Fig. 3 Design?configuration for the precompensation methodology.

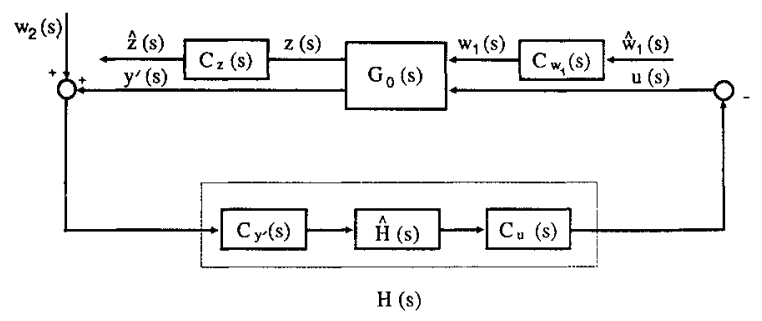

Fig. 4 Implementation configuration for the precompensation methodology.

Likewise $\left(A, B, C, D_{1}, E_{1}\right)$ is said to be the state representation of Eq. (56) if Eqs. (53-55) hold.

Now, for a nominal value $k_{\text {nom }}$ of the spring stiffness let the corresponding state representation of the benchmark system shown in Fig. 2 be given by

$$
\begin{gathered}
\dot{x}(t)=A_{0}\left(k_{\mathrm{nom}}\right) x(t)+B_{0} u(t)+D_{1,0} w_{1}(t) \\
z(t)=E_{1,0} x(t) \\
y^{\prime}=z(t)
\end{gathered}
$$

Also, let $G_{0}(s)$ be the transfer matrix representation of Eqs. (57-59).

A precompensation strategy was used for control law design. This precompensation strategy is illustrated by Figs. 3 and 4 . As shown in Fig. 3 we simply embed the precompensation filters $C_{u}(s), C_{y^{\prime}}(s), C_{w_{1}}(s)$, and $C_{z}(s)$ in the plant a priori and design the ME/OP controller $\hat{H}(s)$ for this modified design plant. Then, as illustrated in Fig. 4, the precompensation dynamics $C_{u}(s)$ and $C_{y^{\prime}}(s)$ are included in the implemented compensator $H(s)$. The sensor noise $\hat{w}_{2}(s)$ in Fig. 3 is fictitious since it is added to the pseudo-output $\hat{y}^{\prime}(s)$. Its intensity $\hat{V}_{2}$ is chosen to aid in the determination of the controller authority. It is not difficult to show that for both Figs. 3 and 4 the closed-loop transfer function $G_{c \ell}(s)$ satisfying

$$
\left[\begin{array}{c}
z(s) \\
y^{\prime}(s)
\end{array}\right]=\left[G_{c f}(s)\right]\left[\begin{array}{l}
w_{1}(s) \\
u(s)
\end{array}\right]
$$

is identical in Figs. 3 and 4. Hence, this methodology insures that if $\hat{H}(s)$ is stabilizing in the feedback loop of Fig. 3, then $H(s)$ is stabilizing in the feedback loop of Fig. 4. In addition, Eq. (60) also insures that the transfer function between $z(s)$ and $w_{1}(s)$ is preserved, thus insuring the preservation of the attenuation from $w_{1}$ to $z$. This precompensation methodology was used in Ref. 9 to achieve controller roll off and to force the design plant to appear to be rate feedback. Its use for the benchmark problem is detailed later.

To describe the control design process for each controller, assume that $\left(A, B, C, D_{1}, E_{1}\right)$ is the state-space representation corresponding to $\hat{G}(s)$ in Fig. 3, such that

$$
\dot{x}(t)=A x(t)+B \hat{u}(t)+D_{1} \hat{w}_{1}(t)
$$

$$
\begin{gathered}
\hat{z}(t)=E_{1} x(t) \\
\hat{y}^{\prime}(t)=C x(t) \\
\hat{y}(t)=\hat{y}^{\prime}(t)+\hat{w}_{2}(t)
\end{gathered}
$$

is the state-space representation of the design plant. The synthesis of $\hat{H}(s)$ in Fig. 3 was based on the solution of the design equations $(41-46)$.

The state-space basis of Eqs. (61-64) was chosen such that $A$ is block-diagonal with a $2 \times 2$ diagonal block of the form

$$
\left[\begin{array}{cc}
0 & \omega_{0} \\
-\omega_{0} & 0
\end{array}\right]
$$

corresponding to the vibrational mode of the system with nominal natural frequency $\omega_{0}$. System uncertainty was assumed to be in the frequency of this mode, and thus the parameter $r$ in Eqs. (41), (42), and (48) is given by $r=1$. The corresponding uncertainty pattern matrix $A_{1}$ was given by

$$
A_{1}=\text { block-diag }\left(0, \ldots, 0,\left[\begin{array}{cc}
0 & 1 \\
-1 & 0
\end{array}\right], 0, \ldots, 0\right)
$$

where location of the nonzero $2 \times 2$ block corresponded to the location of the dynamics of the vibrational mode in $A$. The design weights $R_{1}, R_{2}, \bar{V}_{1}$, and $V_{2}$ were given by

$$
R_{1}=E_{1}^{T} E_{1}, \quad \bar{V}_{1}=D_{1} D_{1}^{T}, \quad R_{2}=V_{2}=\rho
$$

The design parameters are $\rho$, which determines the control authority, and $\delta_{1}^{1 / 2}$, which weights $A_{1}$ and reflects the level of modal uncertainty. Note that since the stiffness $k$ was assumed to be in the interval $[0.5 \mathrm{~N} / \mathrm{m}, 2.0 \mathrm{~N} / \mathrm{m}]$, the natural frequency $\omega$ of the vibrational mode was in the interval $[1.0$ $\mathrm{rad} / \mathrm{s}, 2.0 \mathrm{rad} / \mathrm{s}]$.

Three controllers are described next. Controllers 1 and 2 were developed to meet the objectives of design problem 1 whereas controller 3 was developed for design problem 2 . Controllers 1 and 3 were formulated as standard $H_{2}$ disturbance rejection problems, i.e., $C_{u}(s)=C_{y^{\prime}}(s)=1$ in Figs. 3 and 4 . The disturbance weighting matrix $C_{w_{1}}(s)$ was chosen in the control design process for controller 3 to reflect knowledge of the sinusoidal nature of the disturbance. For controller 2, precompensation $C_{u}(s)$ was added to nullify the effects of the rigid-body mode in a frequency band approximately one decade above and one decade below the frequency of the vibrational mode. Basically, this was an attempt to make the problem "easier" for the LQG part of the design. As will be shown, $C_{u}(s)$ is simply a second-order lead-lag filter. The "lag" poles were included not only to make the precompensation realizable in state space but also to prevent the LQG segment of the controller from having to provide additional roll off. The description of each controller includes the precompensation dynamics used to develop the design model and the stiffness $k_{\text {nom }}$ of the design model. The parameter $k_{\text {nom }}$ was not chosen to be $1 \mathrm{~N} / \mathrm{m}$ as might be expected because it was experimentally observed that as $\delta_{1}$ increased the closedloop system tended to become robust with respect to positive perturbations in $k_{\text {nom }}$ faster than with respect to negative perturbations.

The settling time for each system was chosen to be the time required for the displacement of mass 2 to reach and stay within the interval $[-0.1 \mathrm{~m}, 0.1 \mathrm{~m}]$. Each of the controllers satisfied the corresponding settling time objectives when connected to the model corresponding to $k=1 \mathrm{~N} / \mathrm{m}$. Also, each of the controllers stabilizes the plant for $k \in[0.5 \mathrm{~N} / \mathrm{m}, \quad 2.0$ $\mathrm{N} / \mathrm{m}]$. To illustrate the effectiveness of maximum entropy design in inducing robustness, each of the three controllers is compared with the LQG design that was used to initialize the design process illustrated by Fig. 1. The gain margin (GM) and 
phase margin (PM) listed for each controller are the margins yielded by implementing each controller with the corresponding design plant. In addition, for controllers 1 and 2 a simulation is provided that shows the mass 2 displacement response when the sensor measurements are corrupted by a noise process $w_{2}(t)$. In these simulations, the noise was chosen to be white with a uniform distribution in the interval $[-0.01 \mathrm{~m}$, $0.01 \mathrm{~m}]$

\section{Controller 1 (for Design Problem 1)}

The parameters for controller 1 are as follows:

$$
\begin{gathered}
C_{u}(s)=C_{y^{\prime}}(s)=C_{w_{1}}(s)=C_{z}(s)=1 \\
\rho=0.00001, \quad \delta_{1}^{1 / 2}=0.2 \\
k_{\text {nom }}=0.6 \mathrm{~N} / \mathrm{m} \Rightarrow \omega_{0}=1.0954 \mathrm{rad} / \mathrm{s}
\end{gathered}
$$

order of $\hat{G}(s)=$ order of $\hat{H}(s)=4 \Rightarrow$ full-order design settling time of the mass 2 displacement $=15 \mathrm{~s}$ (for $k=1)$ peak response of the mass 2 displacement $=0.7 \mathrm{~m}$ (for $k=1$ )

$H(s)=\frac{194390(s+0.36679)\left[(s-0.11735)^{2}+0.90996^{2}\right]}{(s+81.438)(s+131.04)\left[(s+2.9049)^{2}+1.8615^{2}\right]}$

Controller 1: stable for $0.45 \leq k \leq 2.05, \mathrm{GM}=3 \mathrm{~dB}$, $\mathrm{PM}=10 \mathrm{deg}$

LQG controller: stable for $0.59 \leq k \leq 1.06, \mathrm{GM}=1 \mathrm{~dB}$, PM $<1$ deg

The impulse responses of the mass 2 displacement (the output performance variable), the mass 1 displacement, and the control signal are shown, respectively, in Figs. 5-7 for $k=1.0$, 0.5 , and $2.0 \mathrm{~N} / \mathrm{m}$. The impulse response of the displacement of mass 2 for $k=1.0 \mathrm{~N} / \mathrm{m}$ with noise-corrupted measurements is shown in Fig. 8. The Nyquist plot of the loop transfer function is shown in Fig. 9

\section{Controller 2 (for Design Problem 2)}

The parameters for controller 2 are as follows:

$$
\begin{gathered}
C_{u}(s)=\frac{100\left[(s+0.04)^{2}+0.0693^{2}\right]}{(s+10)^{2}+17.3205^{2}} \\
C_{y^{\prime}}(s)=C_{w_{1}}(s)=C_{z}(s)=1 \\
\rho=0.0001, \quad \alpha=0.2 \\
k_{\text {nom }}=0.6 \mathrm{~N} / \mathrm{m} \Rightarrow \omega_{0}=1.0954 \mathrm{rad} / \mathrm{s}
\end{gathered}
$$

order of $\hat{G}(s)=6$, order of $\hat{H}(s)=4 \Rightarrow$ reduced-order design settling time of the mass 2 displacement $=5 \mathrm{~s}$ (for $k=1$ )

peak response of the mass 2 displacement $=0.2 \mathrm{~m}$ (for $k=1$ )
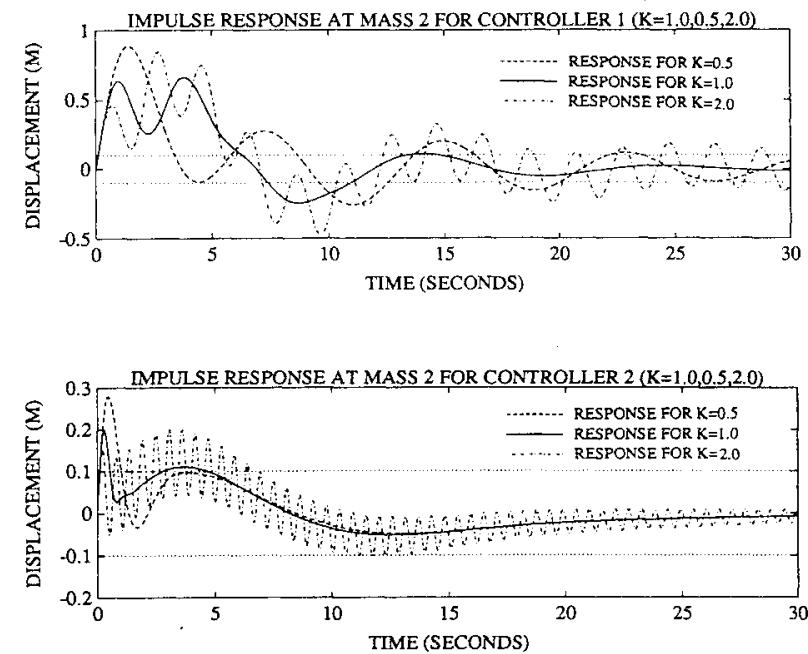

Fig. 5 Mass 2 displacement response to an impulse disturbance for controller 1 (top) and controller 2 (bottom).
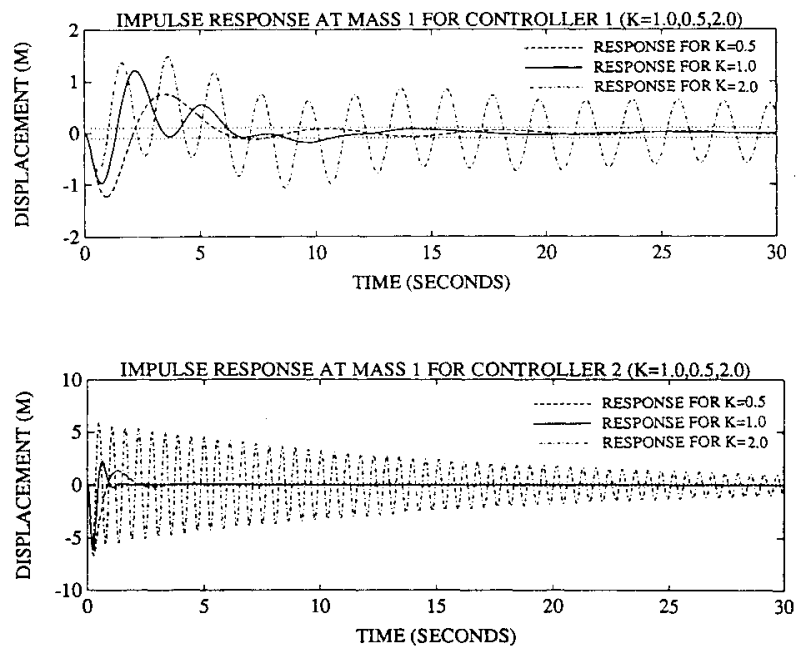

Fig. 6 Mass 1 displacement response to an impulse disturbance for controller 1 (top) and controller 2 (bottom).

The impulse responses of the mass 2 displacement (the output performance variable), the mass 1 displacement, and the control signal are shown, respectively, in Figs. 5-7 for $k=1.0$, 0.5 , and $2.0 \mathrm{~N} / \mathrm{m}$. The impulse response of the displacement of mass 2 for $k=1.0 \mathrm{~N} / \mathrm{m}$ with noise-corrupted measurements is shown in Fig. 8. The Nyquist plot of the loop transfer function is shown in Fig. 10.

\section{Controlier 3 (for Design Problem 2)}

The parameters for controller 3 are as follows:

$$
\begin{gathered}
C_{u}(s)=C_{y},(s)=C_{z}(s)=1, \quad C_{w_{1}}(s)=\frac{1}{(s+0.00050)^{2}+0.50^{2}} \\
k_{\text {nom }}=0.75 \mathrm{~N} / \mathrm{m} \quad \Rightarrow \quad \omega_{0}=1.2247 \mathrm{rad} / \mathrm{s}
\end{gathered}
$$

$$
H(s)=\frac{2490300(s+0.93838)\left[(s+0.30989)^{2}+0.40237^{2}\right]\left[(s+0.040000)^{2}+0.069282^{2}\right]}{(s+54.835)(s+18.831)\left[(s+10.000)^{2}+17.321^{2}\right]\left[(s+0.014561)^{2}+0.031241^{2}\right]}
$$

Controller 2: stable for $0.12 \leq k \leq 2.03, \mathrm{GM}=6 \mathrm{~dB}$, $\mathrm{PM}=33 \mathrm{deg}$

LQG controller: stable for $0.45 \leq k \leq 1.35, \mathrm{GM}=7 \mathrm{~dB}$ $\mathrm{PM}=35 \mathrm{deg}$ 

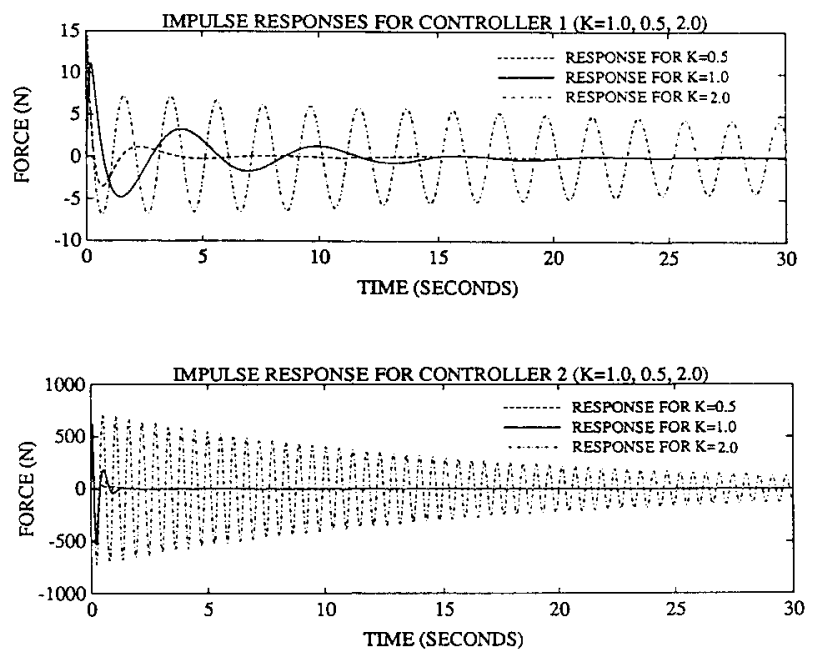

Fig. 7 Control signal response to an impulse disturbance for controller 1 (top) and controller 2 (bottom).
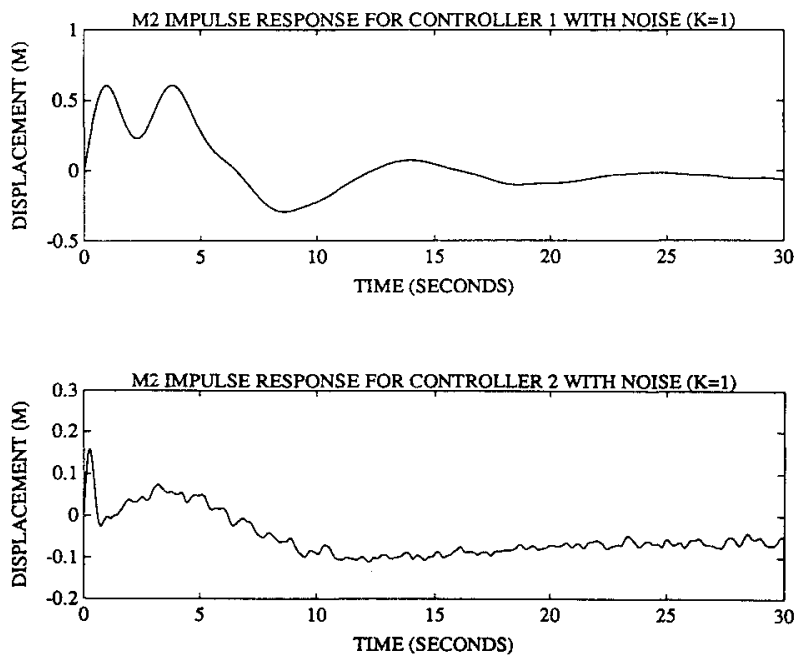

Fig. 8 Mass 2 displacement response to an impulse disturbance with sensor noise for controller 1 (top) and controller 2 (bottom).

order of $\hat{G}(s)=$ order of $\hat{H}(s)=6 \Rightarrow$ full-order design

settling time of the mass 2 displacement $=12 \mathrm{~s}$ (for $k=1$ )

peak response of the mass 2 displacement $=4.4 \mathrm{~m}$

(for $k=1$ )

$$
H(s)=\frac{86816(s+0.12320)\left[(s-0.21281)^{2}+0.96330^{2}\right]\left[(s+0.023916)^{2}+0.42427^{2}\right]}{(s+253.19)(s+38.684)\left[(s+2.5068)^{2}+1.6776^{2}\right]\left[(s+0.0011218)^{2}+0.50138^{2}\right]}
$$

Controller 3: stable for $0.48 \leq k \leq 2.50, \mathrm{GM}=5 \mathrm{~dB}$, $\mathrm{PM}=22 \mathrm{deg}$

LQG controller: stable for $0.43 \leq k \leq 0.78, \mathrm{GM}=4 \mathrm{~dB}$, $\mathrm{PM}=22 \mathrm{deg}$

The responses of the mass 2 displacement (the output performance variable), the mass 1 displacement, and the control signal to a sinusoidal disturbance of frequency of $0.5 \mathrm{rad} / \mathrm{s}$ are shown, respectively, in Figs. $11-13$ for $k=1.0,0.5$, and 2.0 $\mathrm{N} / \mathrm{m}$. It should be noted that no attempt was made to reduce the order of this controller.

\section{Discussion of Results}

Controllers 1 and 2 both satisfied the settling time objectives of design problem 1 but differed significantly in their basic

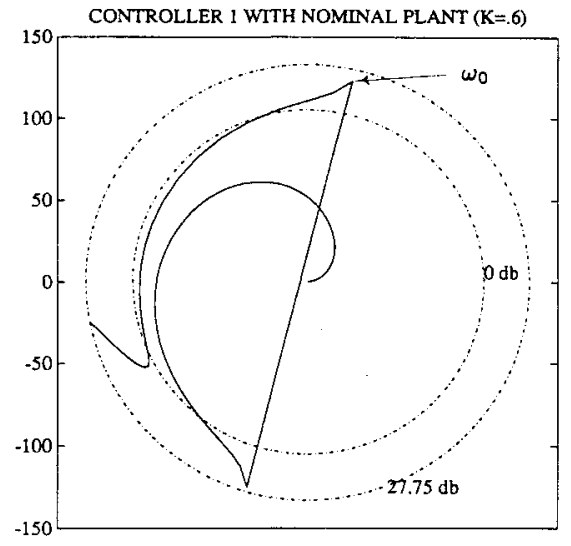

Fig. 9 Nyquist plot of loop transfer function for controller 1.

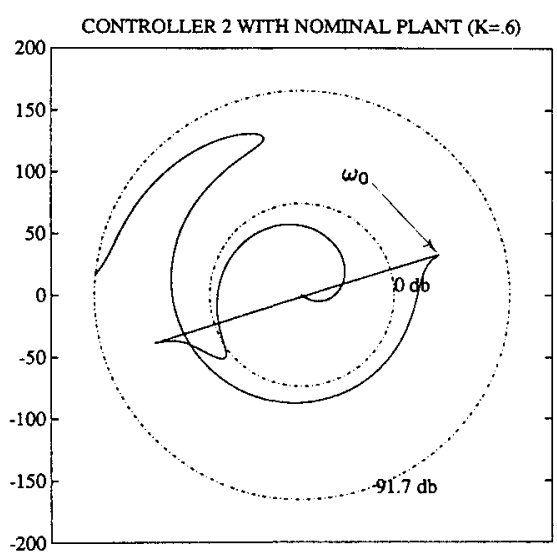

Fig. 10 Nyquist plot of loop transfer function for controller 2.

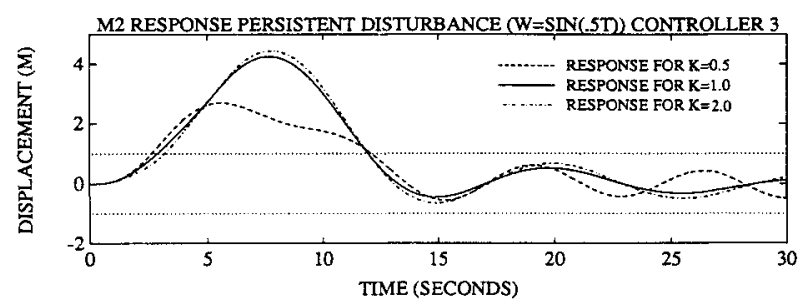

Fig. 11 Mass 2 displacement response to a $0.5 \mathrm{rad} / \mathrm{s}$ sinusoidal disturbance for controller 3 . structure and overall performance. One of the primary differences between these two controllers is best illustrated by the Nyquist diagrams presented in Figs. 9 and 10 of the corresponding loop transfer functions. In these figures it is seen that both controllers placed the loop transfer function in the first quadrant just before the nominal frequency $\omega_{0}$. This insured stability when $180 \mathrm{deg}$ of phase lag was added due to the undamped vibrational mode of the plant. However, despite this similarity, the route that the controllers took to place the loop transfer function in the first quadrant was vastly different. As seen in Fig. 9, controller 1 chose to force the loop transfer function to reach the first quadrant via the second quadrant. This required substantial phase lag from the compensator. However, compensator gain was also needed to achieve performance. Hence, controller 1 included a complex 


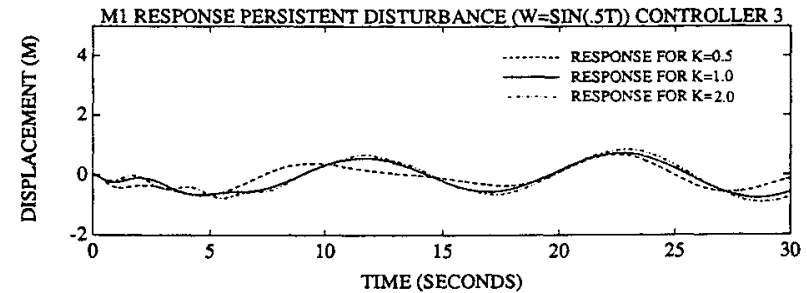

Fig. 12 Mass 1 displacement response to a $0.5 \mathrm{rad} / \mathrm{s}$ sinusoidal disturbance for controller 3 .

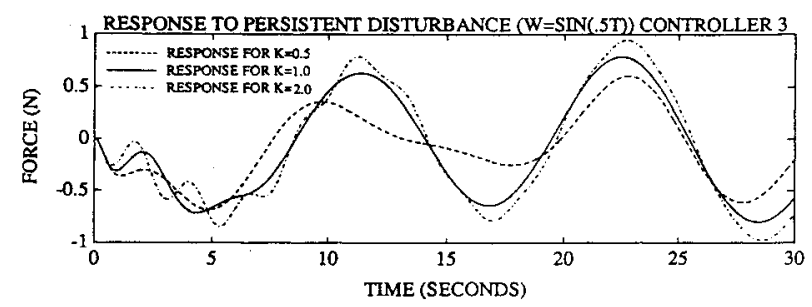

Fig. 13 Control signal response to a $0.5 \mathrm{rad} / \mathrm{s}$ sinusoidal disturbance for controller 3 .

pair of nonminimum phase zeros to achieve both phase lag and increased gain. On the other hand, the precompensation chosen for controller 2 was such that, as shown in Fig. 10, the loop transfer function reached the first quadrant via the third and fourth quadrants. The desired lead and gain increases were accomplished via minimum phase compensator zeros.

Figure 5 compares the impulse response of mass 2 for controllers 1 and 2 whereas Fig. 6 compares the impulse response of mass 1 for the two controllers. It is easily seen from these figures that controller 2 reduced the effects of the impulse disturbance on the system much more than controller 1 . In addition, it is seen in the data listed for each controller that controller 2 has significantly higher gain and phase margins than controller 1 , a feature that might be desirable in some applications. However, Figure 7 reveals that the greater performance of controller 2 was achieved at the expense of much greater control authority, and Fig. 8 shows that, due to its higher bandwidth, controller 2 yields a closed-loop system that is much more sensitive to sensor noise. In fact, the control authority required by controller 2 would likely require an actuator with mass many times the total mass of the original system. Controllers 1 and 3 avoided the use of an impractical amount of actuator authority by the judicious use of nonminimum phase zeros. We conjecture that a low authority controller that meets the performance objectives cannot be designed for the benchmark problem without using nonminimum phase zeros.

It is interesting to note that the data for controller 2 shows that the initiating LQG controller has larger gain and phase margins than the robust controller but also has less robustness with respect to the uncertainty in the stiffness $k$. This provides an illustration of a control problem in which the gain and phase margins do not necessarily imply parametric robustness.

The data for each of the three controllers clearly show that the initializing LQG designs did not satisfy the robustness requirements with respect to the uncertainty in $k$. The efficacy of maximum entropy design in providing the needed robustness was thus clearly illustrated by the results of this paper.

\section{Conclusion}

This paper has demonstrated the application of the maximum entropy/optimal projection methodology for designing robust reduced-order controllers to a benchmark problem. The use of maximum entropy uncertainty modeling clearly provided the needed robustness that was not achievable by simple LQG control. The results of this paper also illustrate the use of a precompensation methodology that essentially allows the control designer to precondition the design plant by embedding in this plant judiciously designed filters. These filters are then included in the implemented compensator. The order of the controller designed using the precompensation methodology was reduced using optimal projection theory.

\section{Acknowledgments}

This work was supported by the Air Force Office of Scientific Research under Contracts F49620-89-C-0011 and F4962089-C-0029.

\section{References}

l Junkins, J. L. (ed.), Mechanics and Control of Large Flexible Structures, Vol. 129, Progress in Astronautics and Aeronautics, AIAA, Washington, DC, 1990.

${ }^{2}$ Hyland, D. C., "Optimal Regulation of Structural Systems with Uncertain Parameters,' MIT Lincoln Lab., TR-551, DDC\# ADA$099111 / 7$, Lexington, MA, Feb. 2, 1991.

${ }^{3}$ Hyland, D. C., "Minimum Information Stochastic Modeling of Linear Systems with a Class of Parameter Uncertainties," Proceedings of the American Control Conference, Arlington, VA, June 1982, pp. $620-627$.

${ }^{4}$ Hyland, D. C., "Maximum Entropy Stochastic Approach to Controller Design for Uncertain Structural Systems," Proceedings of the American Control Conference, Arlington, VA, June 1982, pp. 680688 .

${ }^{5}$ Hyland, D. C., "Mean-Square Optimal, Full-Order Compensation of Structural Systems with Uncertain Parameters," MIT Lincoln Lab., TR-626, Lexington, MA, June 1, 1983.

${ }^{6}$ Hyland, D. C., and Madiwale, A. N., "A Stochastic Design Approach for Full-Order Compensation of Structural Systems with Uncertain Parameters,"' Proceedings of the AIAA Guidance and Control Conference, AIAA, New York, 1981, pp. 324-332.

${ }^{7}$ Phillips, D. J., Hyland, D. C., and Collins, E. G., Jr., "Experimental Demonstrations of Active Vibration Control of Flexible Structures," Proceedings of the IEEE Conference on Decision and Control, Honolulu, HI, Dec. 1990, pp. 2024-2029.

${ }^{8}$ Collins, E. G., Phillips, D. J., and Hyland, D. C., "Robust Decentralized Control Laws for the ACES Structure," Control Systems Magazine, Vol. 11, No. 3, April 1991, pp. 62-70.

${ }^{9}$ Collins, E. G., Jr., King, J. A., and Phillips, D. J., "High Performance, Accelerometer-Based Control of the Minimast Structure," Journal of Guidance, Control, and Dynamics (to be published).

${ }^{10}$ Gruzen, A., "Robust Reduced Order Control of Flexible Structures," C. S. Draper Lab., Rept. CSDL-T-900, Cambridge, MA, April 1986.

${ }^{11}$ Gruzen, A., and Vander Velde, W. E., "Robust Reduced Order Control of Flexible Structures Using the Optimal Projection/Maximum Entropy Design Methodology," AIAA Guidance, Navigation, and Control Conference, Williamsburg, VA, Aug. 1986.

${ }^{12}$ Cheung, M., and Yurkovich, S., "On the Robustness of MEOP Design Versus Asymptotic LQG Synthesis," IEEE Transactions on Automatic Control, Vol. 33, No. 11, 1988, pp. 1061-1065.

${ }^{13}$ Hyland, D. C., and Bernstein, D. S., "The Optimal Projection Equations for Fixed-Order Dynamic Compensation,' IEEE Transactions on Automatic Control, Vol. AC-29, No. 11, 1984, pp. 10341037.

${ }^{14}$ Bernstein, D. S., "Robust Static and Dynamic Output-Feedback Stabilization: Deterministic and Stochastic Perspectives," IEEE Transactions on Automatic Control, Vol. AC-32, No. 12, 1987, pp. 1076-1084.

${ }^{15}$ Bernstein, D. S., and Haddad, W. M., “Robust Stability and Performance via Fixed-Order Dynamic Compensation with Guaranteed Cost Bounds," Mathematics of Control, Signals, and Systems, Vol. 3, No. 3, 1990, pp. 139-163.

${ }^{16}$ Bernstein, D. S., and Haddad, W. M., "The Optimal Projection Equations with Petersen-Hollot Bounds: Robust Stability and Performance via Fixed-Order Dynamic Compensation for Systems with Structured Real-Valued Parameter Uncertainty," IEEE Transactions on Automatic Control, Vol, 33, No. 6, 1988, pp. 578-582.

${ }^{17}$ Bernstein, D. S., and Haddad, W. M., "LQG Control with an $H_{\infty}$ Performance Bound: A Riccati Equation Approach," IEEE Transactions on Automatic Control, Vol. 34, No. 3, 1989, pp. 293-305.

${ }^{18}$ Bernstein, D. S., and Hyland, D. C., "The Optimal Projection Approach to Robust, Fixed-Structure Control Design," Mechanics and Control of Large Space Structures, edited by J. L. Junkins, Vol. 129, Progress in Astronautics and Aeronautics, AIAA, Washington, 
D.C., pp. 237-293.

${ }^{19}$ Wie, B., and Bernstein, D. S., "A Benchmark Problem for Robust Control Design," Proceedings of the American Control Conference, San Diego, CA, May 1990, pp. 961-962.

${ }^{20}$ Greeley, S. W., and Hyland, D. C., "Reduced-Order Compensation: Linear-Quadratic Reduction Versus Optimal Projection," Journal of Guidance, Control, and Dynamics, Vol. 11, No. 4, 1988, pp. $328-335$.

${ }^{21}$ Richter, S., and Collins, E. G., Jr., “A Homotopy Algorithm for Reduced-Order Controller Design Using the Optimal Projection Equations," Proceedings of the IEEE Conference on Decision and Control, Tampa, FL, Dec. 1989, pp. 506-511.

${ }^{22}$ Hyland, D. C., and Richter, S., "On Direct Versus Indirect Methods for Reduced-Order Controller Design," IEEE Transactions on Automatic Control, Vol. 35, No. 3, 1990, pp. 377-379.

${ }^{23}$ Bernstein, D. S., and Haddad, W. M., "Robust Stability and Performance Analysis for State Space Systems via Quadratic Lyapunov Bounds," SIAM Journal on Matrix Analysis and Applications, Vol. 11, No. 2, 1990, pp. 239-271.

${ }^{24}$ Chang, S. S. L., and Peng, T. K. C., "Adaptive Guaranteed Cost Control of Systems with Uncertain Parameters," IEEE Transactions on Automatic Control, Vol. AC-17, No. 4, 1972, pp. 474-483.

${ }^{25}$ Petersen, I. R., "A Riccati Equation Approach to the Design of Stabilizing Controllers and Observers for a Class of Uncertain Linear Systems," IEEE Transactions on Automatic Control, Vol. AC-30, No. 9, 1985, pp. 904-907.

${ }^{26}$ Petersen, I. R., and Hollot, C. V., "A Riccati Equation Approach to the Stabilization of Uncertain Systems," Automatica, Vol 22, No. 4, 1986, pp. 397-411.
${ }^{27}$ Khargonekar, P. P., Petersen, I. R., and Zhou, K., "Robust Stabilization of Uncertain Linear Systems: Quadratic Stabilizability and $H^{\infty}$ Control Theory," IEEE Transactions on Automatic Control, Vol. 35, No. 3, 1990, pp. 356-361.

${ }^{28} \mathrm{Haddad}$, W. M., and Bernstein, D. S., "Robust Stability and Performance Analysis for State Space Systems Via Parameter-Dependent Lyapunov Functions: The Popov Criterion Revisited," Proceedings of the IEEE Conference on Decision and Control, Brighton, England, UK, Dec. 1991

${ }^{29}$ Hall, S. R., MacMartin, D. G., and Bernstein, D. S., "Incoherence, Equipartition, and Covariance Averaging in the Analysis of Uncertain Systems" submitted to IEEE Transactions on Automatic Control.

${ }^{30}$ Collins, E. G., Jr., and Richter, S., "A Homotopy Algorithm for Synthesizing Robust Controllers for Flexible Structures Via the Maximum Entropy Design Equations," Third Air Force/NASA Symposium on Recent Advances in Multidisciplinary Analysis and Optimization, San Francisco, CA, Sept. 1990, pp. 324-333.

${ }^{31}$ Yousuff, A., and Skelton, R. E., "A Note on Balanced Controller Reduction," IEEE Transactions on Automatic Control, Vol. AC-29, No. 3, 1984, pp. 254-256.

${ }^{32}$ Collins, E. G., Jr., and Bernstein, D. S., “Robust Control Design for a Benchmark Problem Using a Structured Covariance Approach," Proceedings of the American Control Conference, San Diego, CA, May 1990, pp. 970, 971.

${ }^{33}$ Collins, E. G., Jr., King, J. A., and Bernstein, D. S., "Robust Control Design for the Benchmark Problem Using the Maximum Entropy Approach," Proceedings of the American Control Conference, Boston, MA, June 1991, pp. 1935, 1936

\section{Fundamentals of Tactical and Strategic Missile Guidance}

By: Paul Zarchan

October 21-23, 1992

Washington, DC

Interceptor guidance system technology is presented in common language using nonintimidating mathematics, arguments, and examples.

Topics include: Important closed form solutions and their unity, comparisons with pursuit guidance, how to construct an adjoint mathematically \& practically, how to use adjoints to analyze missile guidance systems, noise analysis and how to interpret Monte Carlo results, proportional navigation and miss distance, digital noise filters in the homing loop, how to derive optimal guidance laws without optimal control theory, a simple Kalman filter that really works, extended Kalman filtering, Lambert guidance, tactical zone, and much more.

For additional information, FAX or call David Owens,

Continuing Education Coordinator Tel.202/646-7447 FAX 202/646-7508

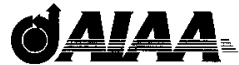

American Institute of Aeronautics and Astronautics Continuing Education Department The Aerospace Center 370 L'Enfant Promenade, SW Washington, DC 20024-2518 